\title{
Hyperhomocysteinemia in Recurrent pregnancy loss
}

\author{
Nisha Bhatia ${ }^{1 *}$, Hemanshu B. ${ }^{2}$ \\ ${ }^{1}$ Department of Obstetrics and Gynecology, Apollo institute of Medical Sciences and Research, Hyderabad, India \\ ${ }^{2}$ Department of Cardiology, Maxcure Hospital, Hyderabad, India
}

Received: 16 April 2017

Accepted: 18 May 2017

*Correspondence:

Dr. Nisha Bhatia,

E-mail: nish_178@yahoo.co.in

Copyright: ( ) the author(s), publisher and licensee Medip Academy. This is an open-access article distributed under the terms of the Creative Commons Attribution Non-Commercial License, which permits unrestricted non-commercial use, distribution, and reproduction in any medium, provided the original work is properly cited.

\begin{abstract}
Background: Hyperhomocysteinemia is associated with many pregnancy related complications. Its association with recurrent pregnancy loss has been investigated recently. Not only it is associated with recurrent pregnancy loss but also associated with complications like Preeclampsia, intrauterine growth retardation and low birth weight. The objective of this study to assess the prevalence of Hyperhomocysteinemia in patients with unexplained recurrent pregnancy loss and to evaluate the association of hyperhomocysteinemia with pregnancy complications in patients of recurrent pregnancy loss.

Methods: 50 Patients of unexplained recurrent pregnancy loss were selected as cases and 50 patients with atleast one successful pregnancy were taken as controls. Their blood sample was assayed for Fasting Homocysteine levels. They were followed up throughout pregnancy for any complications and neonatal outcome was recorded.

Results: 19 patients out of the 50 cases had Hyperhomocysteinemia. Hyperhomocysteinemia was thrice more common in Primary aborters when compared to secondary aborters. Out of 19 patients with hyperhomocysteinemia in patients with recurrent pregnancy loss, 14 patients also developed Hypertensive disorder of pregnancy during their antenatal period. Among patients with Hyperhomocysteinemia in study group, $47 \%$ had low birthweight babies when compared to $25 \%$ among control group.

Conclusions: Hyperhomocysteinemia is associated with recurrent pregnancy loss and patients of Recurrent pregnancy loss who have Hyperhomocysteinemia have a higher risk of developing pregnancy complications like Preeclampsia, low birth weight and Intrauterine growth retardation.
\end{abstract}

Keywords: Hyperhomocysteinemia, Hypertensive disorders, Intrauterine growth restriction, Low birth weight, Plasma homocysteine, Primary aborter

\section{INTRODUCTION}

Recurrent pregnancy loss (RPL) is traditionally defined as the occurrence of three or more pregnancy losses before 20 weeks of gestation. ${ }^{1}$ Clinical investigations may be initiated after two spontaneous abortions. Cause remains unknown in approximately $50 \%$ of the cases of RPL. Hyperhomocysteinemia defined as Plasma Homocysteine levels more than $95^{\text {th }}$ percentile from baseline. Studies done in last two decades have found that the mean fasting level of Plasma homocysteine is higher in women with recurrent pregnancy loss. ${ }^{2}$ The basic mechanism of Hyperhomocysteinemia is atherosclerosis and thromboembolism. Trophoblastic cell death due to oxidative stress and apoptosis may be responsible for the obstetric complications. ${ }^{2}$ Not only RPL, Hyperhomocysteinemia is associated with many pregnancy related complications. ${ }^{3}$ The present study was done to assess the association of Hyperhomocysteinemia in recurrent pregnancy loss in our population. 
The primary aim of this study is to assess the prevalence of Hyperhomocysteinemia in patients with unexplained recurrent pregnancy loss. The secondary outcome of this study is to evaluate the association of hyperhomocysteinemia with pregnancy induced hypertension, intrauterine growth restriction and low birth weight in patients with recurrent pregnancy loss.

\section{METHODS}

It was a prospective study conducted in Government Maternity Hospital, Sultan Bazaar, from 2007 to 2009 over two years. A total no: of 50 patients of unexplained recurrent pregnancy loss were taken as study and 50 patients with atleast one successful pregnancy were taken as controls. Controls were comparable in age, residence and socioeconomic status.

\section{Exclusion criteria}

Subjects were excluded from study group if they had any anatomical abnormalities, women with any history of vascular thrombotic events, or any immunological disorders or endocrinological disorders or infective causes of recurrent pregnancy loss or if they gave history of vitamin B6 supplementation or folic acid within 6 months of study period, history of any other medical or endocrine disorder.

The subjects who met the inclusion criteria were included in the first trimester in the study after informed consent. A venous sample of blood by venipuncture in antecubital vein in order to assay Fasting plasma Homocysteine. The collected sample was assayed in 4 hours of sample collection by Chemiluminiscence immunoassay. Hyperhomocysteinemia is defined as plasma levels of Homocysteine more than the 95th percentile. In Indian studies, Yajnik et al and Kumar et al have taken cut off in pregnancy as $10.5 \mu \mathrm{mol}$. ${ }^{4,5}$ Accordingly, in present study we have also taken the cut off as $10.5 \mu \mathrm{mol}$. These subjects were given the same standard of care as other antenatal patients with regular antenatal check-ups and iron and folic acid supplements as routinely advised. They were monitored for development of any antenatal complications such as early pregnancy loss, Preeclampsia or Intrauterine growth restriction and were followed till the time of delivery. Condition of baby at birth including weight, APGAR score, requirement of NICU were used as markers for perinatal outcome.

\section{Statistical analysis}

Statistical Analysis was done using SPSS data packages. Chi square test and Fischer tests were applied wherever required.

\section{RESULTS}

A total of 100 subjects were included in the study. 50 were study and 50 were controls. Among 50 patients of recurrent pregnancy loss, $58 \%$ belonged to the age group of more than 25 years. The mean age of recurrent pregnancy loss in our study was $26.226 \pm 4.882$ years. When the risk factors for hyperhomocysteinemia were evaluated it was noted that Tobacco chewing and Passive smoking had significant correlation with hyperhomocysteinemia ( $\mathrm{p}$ value 0.04 ).

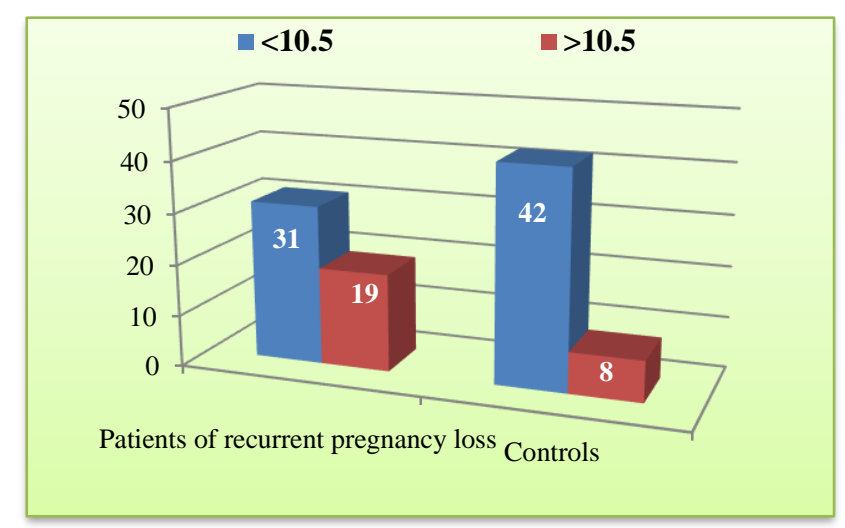

Figure 1: Plasma homocysteine levels in patients of recurrent pregnancy loss versus controls.

Among 50 patients in the study group, 19 patients had hyperhomocysteinemia when compared to only 8 patients in the controls that showed a statistically significant association of hyperhomocysteinemia with recurrent pregnancy loss ( $p$ value $<0.01$ ) (Figure1).

The mean value of homocysteine levels in the study group was $11.335 \pm 5.054 \mu \mathrm{mol} / \mathrm{dl}$ whereas in controls it was $7.654 \pm 3.477 \mu \mathrm{mol} / \mathrm{dl}$. $\mathrm{Z}$ test applied to the means revealed association between recurrent pregnancy loss and Hyperhomocysteinemia (Z-value- 4.254). There was no statistically significant association between hyperhomocystenemia with age or body mass index in both study and control groups.

Table 1: Hyperhomocysteinemia in primary aborters versus secondary aborters.

\begin{tabular}{|llll|} 
Plasma & $\begin{array}{l}\text { Primary } \\
\text { aborters }\end{array}$ & $\begin{array}{l}\text { Secondary } \\
\text { aborters }\end{array}$ & Total \\
\hline$<10.5$ & 10 & 21 & 31 \\
\hline$>10.5$ & 14 & 5 & 19 \\
\hline & 24 & 26 & 50 \\
\hline
\end{tabular}

Table 2: Hyperhomocysteinemia and pregnancy outcome.

\begin{tabular}{|lllll|}
\hline $\begin{array}{l}\text { Plasma } \\
\text { homocysteine }\end{array}$ & Abortion & $\begin{array}{l}\text { Vaginal } \\
\text { delivery }\end{array}$ & $\begin{array}{l}\text { Cesarean } \\
\text { section }\end{array}$ & Total \\
\hline$<10.5$ & 1 & 12 & 18 & 31 \\
\hline$>10.5$ & 3 & 4 & 12 & 19 \\
\hline & 4 & 16 & 30 & 50 \\
\hline
\end{tabular}

The study population consisted of 24 primary aborters (subjects with recurrent abortions without any successful 
pregnancy) and 26 secondary aborters. (subjects with recurrent abortions after at least one successful pregnancy) Hyperhomocysteinemia was thrice more common in primary aborters when compared to secondary aborters ( $\mathrm{p}$ value; <0.01).

The Odds of Abortion was 2.21 times higher among patients with Hyperhomocysteinemia when compared to patients with normal Homocysteine levels.

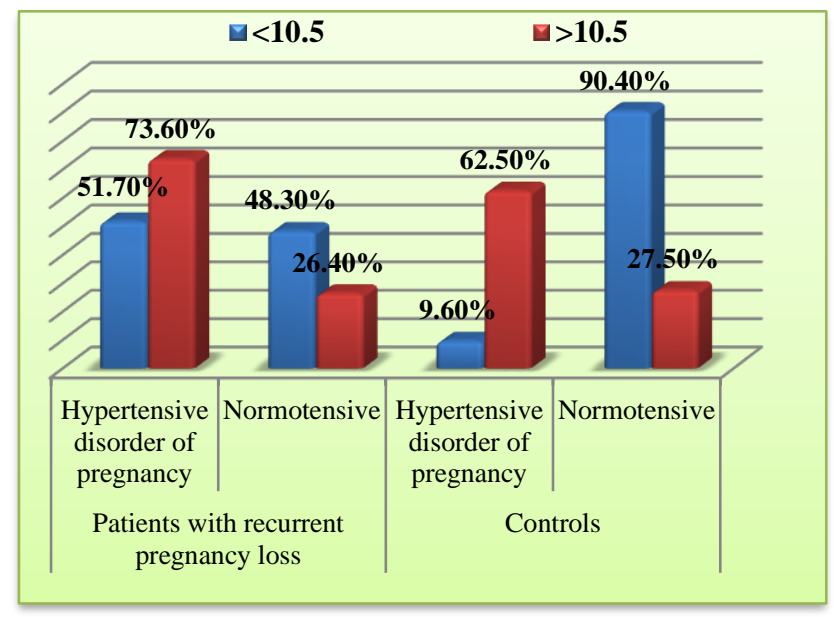

Figure 2: Hyperhomocysteinemia and hypertensive disorders in pregnancy in patients of recurrent pregnancy loss versus controls.

Out of 19 patients with hyperhomocysteinemia in patients with recurrent pregnancy loss, 14 patients also developed Hypertensive disorder of pregnancy during their antenatal period. Similar statistical association was seen in the control group where 5 out of 8 patients of Hyperhomocysteinemia developed Hypertension in pregnancy, showing significant association of Hyperhomocysteinemia with hypertensive disorder of pregnancy (Figure 2).

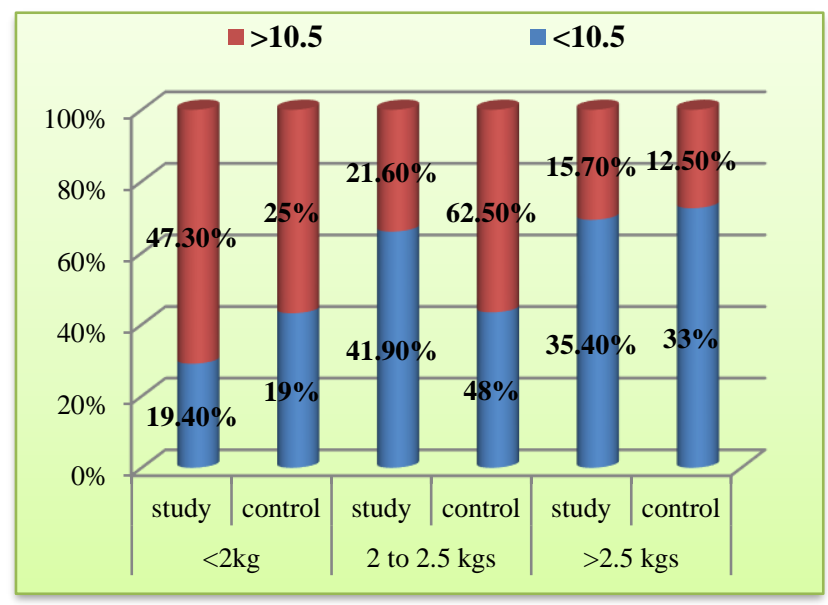

Figure 3: Hyperhomocysteinemia and low birth weight among patients with recurrent pregnancy loss versus controls
Among patients with Hyperhomocysteinemia in study group, $47 \%$ had low birthweight babies when compared to $25 \%$ among control group ( $\mathrm{p}$ value -0.0227 ). Due to increased morbidity in patients of hyperhomocysteinemia such as preeclampsia, growth restriction ( $\mathrm{p}$ value 0.003 ) and low birth weight (p-value 0.02), the chances of Cesarean section was also more in these patients ( $\mathrm{p}$-value 0.03) (Figure 3).

\section{DISCUSSION}

Recurrent Pregnancy loss has been traditionally defined as occurrence of three or more pregnancy losses with an incidence of around $1 \%{ }^{1}$ In about $50 \%$ of patients of RPL cause remains unknown., ${ }^{2,3}$ Hyperhomocysteinemia has emerged as a strong risk factor for many diseases but its role in RPL has been confirmed recently. ${ }^{2}$ During pregnancy, the levels of plasma homocysteine tends to fall due to increased methionine requirement by fetus, hemodilution and increased renal clearance of homocysteine. Hence the value tends to fall from $15 \mu \mathrm{mol}$ to $10.5 \mu \mathrm{mol}^{4}$ Homocysteine Studies conducted in pregnancy by Fatini etal and Yajnik et al have taken 10.5 $\mu \mathrm{mol}$ as the cutoff in pregnancy. ${ }^{2,3}$ In present study, Hyperhomocysteinemia has been defined as more than $10.5 \mu \mathrm{mol} / \mathrm{dl}$. Nelen et al did a study to evaluate the risk factors for Hyperhomocysteinemia. ${ }^{5} \mathrm{He}$ found no significant correlation between age and body mass index in recurrent pregnancy loss and hyperhomocysteinemia. Similarly, present study also did not find any such association. Tobacco chewing and passive smoking were not evaluated in their study but in present study, there was a statistically significant association with hyperhomocysteinemia and recurrent pregnancy loss. Similar to the present study, Nelen et al found Hyperhomocysteinemia more common in primary aborters while Comans et al and Wouters et al found Hyperhomocysteinemia more common in secondary aborters. ${ }^{5-7}$ The mechanism could be an intrinsic genetic metabolic disorder in primary aborters rather than dietary and environmental factors that could have a role in secondary aborters.

The prevalence of Hyperhomocysteinemia in RPL in present study was $38 \%$ and the mean value of homocysteine level was $11.335 \mu \mathrm{mol} / \mathrm{dl}$. In most of the western countries the prevalence was around 28 to $33 \%$. In a study by Bianco D et al in Italy among 40 patients of recurrent pregnancy loss, 25\% had hyperhomocysteinemia. ${ }^{8}$ There was also marked variation in the median value in pregnancy from $8.95 \mu$ mol 68 in an Indian study to $13.1 \mu \mathrm{mol} / \mathrm{dl}$ in study by Nelen et $\mathrm{al}^{5}$. The reasons for these inconsistencies can be that there is no homogenous definition of Hyperhomocysteinemia in pregnancy. Etiology of Hyperhomocystenemia is multifactorial, depends on genotype of an individual, has multiple gene gene interactions and also depends on dietary habits and personal habits of an individual. ${ }^{9}$ Despite these inconsistencies, the association of Hyperhomocysteinemia with RPL has been proven. 
Hyperhomocysteinemia not only hampers chances of pregnancy but also interferes with successful pregnancy outcome. ${ }^{10}$ Present study also found a statistically significant association of Hyperhomocysteinemia with complications like early pregnancy loss, preeclampsia, intrauterine growth restriction and low birth weight not only in patients of RPL but also in healthy controls. Hence, we can infer that Plasma Homocysteine measured in early pregnancy may be a positive predictor of pregnancy complications and timely interventions to prevent progression to severe hyperhomocysteinemia may prevent the development of these complications.

\section{CONCLUSION}

The mean plasma homocysteine levels in patients of RPL is 1.5 times higher than in patients with good pregnancy outcome. Homocysteine levels more than $10.5 \mu \mathrm{mol} / \mathrm{dl}$ is not only a risk factor for pregnancy loss but also a predictor of complications like Preeclampsia and IUGR. This warrants lifestyle changes and interventions in prepregnancy and early pregnancy period with diet rich in fruits and vegetables and nutritional supplements containing high folic acid, vitamin B12 and B6 to ensure a healthy and successful pregnancy outcome.

\section{Funding: No funding sources}

Conflict of interest: None declared

Ethical approval: The study was approved by the Institutional Ethics Committee

\section{REFERENCES}

1. Carington B, Sacks G, Regan C. Recurrent Miscarriage pathophysiology and outcome. Curr Opin Obstet Gynecol. 2005;17:591-7.

2. Fatini C, Gensini F, Battaglini B, Prisco D, Cellai $\mathrm{AP}$, Fedi $\mathrm{S}$ et al. Angiotensin converting enzyme DD genotype, angiotensin type 1 receptor $\mathrm{Cc}$ genotype and hyperhomocysteinemia increase first trimester fetal loss and susceptibility. Blood Coagul Fibrinolysis. 2000;11:657-62.
3. Yajnik CS, Deshpande SS, Jackson AA, Refsum H, Rao S, Fisher DJ et al. Vitamin B12 and Folate concentrations during pregnancy and insulin resistance in the offspring: the Pune Maternal Nutrition study. Diabetologia. 2008;51:29.

4. Kumar J, Das SK, Sharma P, Karthikeyan G, Ramakrishnan L, Sengupta S. Homocysteine levels are associated with MTHFR A $1298 \mathrm{C}$ polymorphism in Indian population. J Human Genetics. 2005;50:655-63.

5. Nelen WL, Blom HJ, Steegers EA, den Heijer M, Thomas CM, Eskes TK. Homocysteine and Folate levels as risk factors for REPL. Obstet Gynecol. 2000;95(4):519-24.

6. Coumans AB1, Huijgens PC, Jakobs C, Schats R, de Vries JI, van Pampus MG. Haemostatic and metabolic abnormalities in women with unexplained recurrent abortion. Hum Reprod. 1999;14(1):211-4.

7. Wouters MG1, Boers GH, Blom HJ, Trijbels FJ, Thomas CM, Borm GF et al. Hyperhomocystenemia is a risk factor in women with unexplained REPL. Fertil Steril.1993;60(5):820-5.

8. Del Bianco A, Maruotti G, Fulgieri AM, Celeste T, Lombardi L, Amato NA et al. Recurrent spontaneous miscarriages and Hyperhomocysteinemia. Minerva Ginecol. 2004;56(5):379-83.

9. Misra A, Vikram NK, Pandey RM, Dwivedi M, Ahmad FU, Luthra K et al. Hyperhomocysteinemia and intake of folic acid and Vitamin B12 in urban North India. Eur J Nutr. 2002;41:68-77.

10. Vollset SE, Refsum H, Irgens LM Emblem BM, Tverdal A, Gjessing $\mathrm{HK}$ et al. Plasma total homocysteine, pregnancy complications and adverse pregnancy outcome: the Hordland homocysteine study. Am J Clinical Nutr. 2000;71:962-8.

Cite this article as: Bhatia N, Hemanshu B. Hyperhomocysteinemia in Recurrent pregnancy loss. Int J Reprod Contracept Obstet Gynecol 2017;6:2919-22. 